\title{
Revista Colombiana de

\section{Validación del Minnesota Living with Heart Failure questionnaire (MLFHQ) en pacientes con falla cardíaca}

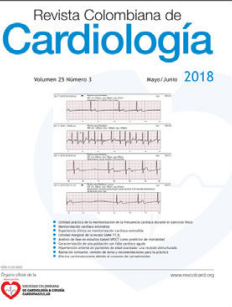

\section{Luz Helena Lugo-Agudelo ${ }^{\mathrm{a}, \mathrm{b}, *}$, Sergio D. Ortiz-Rangel ${ }^{\mathrm{a}, \mathrm{b}}$, Camila Rodríguez-Guevara ${ }^{\mathrm{a}, \mathrm{b}}$, Diana M. Vargas-Montoya ${ }^{\mathrm{b}}$, Daniel Camilo Aguirre-Acevedo ${ }^{a}$, Claudia Y. Vera-Giraldo ${ }^{a}$ y Claudia M. Navas-Ríos ${ }^{a, b}$}

\footnotetext{
a Grupo de Rehabilitación en Salud. Universidad de Antioquia. Clínica Las Américas, Medellín, Colombia

b Unidad de Medicina Física y Rehabilitación, Clínica las Américas, Medellín, Colombia
}

Recibido el 13 de febrero de 2019; aceptado el 1 de abril de 2019

Disponible en Internet el 5 de septiembre de 2019

\author{
PALABRAS CLAVE \\ Calidad de vida; \\ Falla cardíaca; \\ MLHFQ; \\ Validez; \\ Confiabilidad
}

\begin{abstract}
Resumen
Objetivo: validar el cuestionario de calidad de vida "Minnesota Living with Heart Failure" en pacientes con falla cardíaca para su uso en población colombiana.

Materiales y métodos: estudio observacional de validación de una escala, prospectivo en tres momentos: en el primero se hizo la traducción, la retrotraducción y la adaptación cultural; en el segundo se hizo la evaluación de la validez de contenido, constructo, criterio convergente y divergente con el SF36, WHODASII y DASI, y se evaluó la consistencia interna y la fiabilidad intra e interobservador, y en el tercero, longitudinal, se evaluó la sensibilidad al cambio.

Resultados: se incluyeron 134 pacientes, con clase funcional I-IV de la NYHA, fracción de eyección $\leq 50 \%$. El análisis confirmatorio se ajustó a las tres dimensiones de la escala original. Hubo mejor calidad de vida en quienes tuvieron mejor capacidad física de acuerdo con los MET alcanzados. La validez de criterio convergente fue superior a 0,6 y la divergente menor a 0,4 con el SF36, WHODAS II y DASI. Se encontraron diferencias estadísticamente significativas entre el inicio, el primero, el tercero y el sexto mes. El mínimo cambio detectable (MCD) fue para la dimensión física de 6,0, para la dimensión emocional de 4,8 y para el total de 12,6.

Conclusiones: la escala de "Minnesota Living with Heart Failure"' (MLHFQ) es válida, confiable y tiene una buena sensibilidad al cambio para ser utilizada en las personas con falla cardiaca en Colombia.

(c) 2019 Sociedad Colombiana de Cardiología y Cirugía Cardiovascular. Publicado por Elsevier España, S.L.U. Este es un artículo Open Access bajo la licencia CC BY-NC-ND (http:// creativecommons.org/licenses/by-nc-nd/4.0/).
\end{abstract}

\footnotetext{
* Autor para correspondencia.

Correos electrónicos: gruporehabilitacionsalud@udea.edu.co, luzh.lugo@gmail.com (L.H. Lugo-Agudelo).
} 


\section{KEYWORDS}

Quality of life;

Heart failure;

Minnesota Living with

Heart Failure

questionnaire;

MLHFQ;

Validity;

Reliability
Validation of the Minnesota Living with Heart Failure questionnaire in patients with heart failure in Colombia

\begin{abstract}
Objective: To validate the "Minnesota Living with Heart Failure"' quality of life questionnaire in patients with heart failure, for its use in a Colombian population.

Materials and methods: An observational, prospective study with validation of a scale at three points was conducted. First of all, a translation, back-translation, and cultural adaptation were performed. This was followed by the evaluation of the content validity, construct, convergent and divergent criteria with the SF36, WHODAS II and DAS I questionnaires, as well as the assessment of internal consistency and the within- and between-observer reliability. Finally, a longitudinal study was carried out to evaluate the sensitivity to change.

Results: The study included a total of 134 patients, with NYHA functional class I-IV, and an ejection fraction $\leq 50 \%$. The confirmatory analysis was adjusted to the three dimensions of the original scale. There was a better quality of life in those that had a better physical capacity according to the MET achieved. The validity of the convergent criteria was greater than 0.6 , with the divergent being less than 0.4 with the SF36, WHODAS II and DASI. Statistically significant differences were found between the beginning and the first, third, and sixth month. The minimum detectable change was for the physical dimension, 0.6 , for the emotional dimension, 4.8, and for the total, 12.6 .

Conclusions: The "Minnesota Living with Heart Failure" scale is valid and reliable, and has a good sensitivity to change, in order to be used in the populations with heart failure in Colombia. (C) 2019 Sociedad Colombiana de Cardiología y Cirugía Cardiovascular. Published by Elsevier España, S.L.U. This is an open access article under the CC BY-NC-ND license (http:// creativecommons.org/licenses/by-nc-nd/4.0/).
\end{abstract}

\section{Introducción}

La falla cardiaca es un síndrome clínico caracterizado por síntomas como fatiga o disnea de esfuerzo, congestión pulmonar y edema periférico. La enfermedad coronaria es la causa del $70 \%$ de los casos, la enfermedad valvular de un $10 \%$ y las miocardiopatías de otro $10 \%{ }^{1}$.

En el mundo hay entre 23 a 26 millones de personas con falla cardiaca ${ }^{2}$, mientras que en Latinoamérica, la incidencia es de 199/100.000 personas-año; la prevalencia es del $1 \%$, la frecuencia de rehospitalización es del $33 \%$ a los tres meses y la tasa de mortalidad al año de $24,5 \%$. Específicamente en Colombia, se estima una prevalencia de $2,3 \%$, aproximadamente 1.100 .000 personas, de las cuales $59,7 \%$ son hombres y $40,3 \%$ mujeres.

En 2009 la consulta en el servicio de urgencias incrementó en un $50 \%$, en tanto que en los servicios ambulatorios alcanzó un $10 \%$ y en hospitalización por descompensación llegó al $30 \%$, pues pasó de 7.154 a 10.288 casos $^{3}$. El incremento de la prevalencia eleva los costos de su atención, mientras que la falla cardiaca conduce a deterioro funcional y de la calidad de vida ${ }^{4}$.

En un sentido filosófico, calidad de vida es lo que hace que una vida sea mejor, que sea "una buena vida" 5 . La Organización Mundial de la Salud la define en función de la manera en que el individuo percibe el lugar que ocupa en la vida, en el contexto de la cultura y del sistema de valores en que vive, y en relación con sus objetivos, expectativas, normas y preocupaciones. La calidad de vida relacionada con la salud es el componente de la calidad de vida debido a las condiciones de salud de las personas y referido a partir de las experiencias subjetivas de ellas sobre su salud global ${ }^{6,7}$. La evaluación de la calidad de vida, la función, los síntomas y la adherencia al tratamiento son desenlaces centrados en el paciente, claves para evaluar la efectividad de programas e intervenciones en condiciones crónicas como la falla cardíaca $^{8}$. La calidad de vida en personas con falla cardiaca se encuentra afectada por la limitación en la movilidad, dolor, ansiedad y depresión, y su medición se convierte en un predictor independiente de pronóstico, mortalidad, trasplante cardíaco, implante de dispositivo de asistencia ventricular izquierda o reingreso por descompensación?.

Para evaluar la calidad de vida existen instrumentos genéricos, como el SF36 validado en Colombia, y específicos, que tienen en cuenta las condiciones propias de una condición de salud ${ }^{10}$. En la guía colombiana de Falla Cardíaca hay una recomendación fuerte a favor para el uso del cuestionario de Minnesota Living with Heart Failure Questionnaire (MLHFQ) y el Kansas City Cardiomyopathy Questionnaire (KCCQ) $)^{1}$.

La escala MLHFQ fue desarrollada por Rector et al. en 1984 y ha sido traducida a más de 32 lenguas, con buenas propiedades sicométricas ${ }^{11}$. En una revisión sistemática compararon esta escala con otros instrumentos como Chronic Heart Failure Questionnaire (CHFQ), Quality of Life Questionnaire for Severe Heart Failure (QLQ-SHF), Kansas City Cardiomyopathy Questionnaire (KCCQ) and left ventricular dysfunction (LVD-36) y concluyeron que las propiedades psicométricas fueron superiores en el MLHFQ ${ }^{12}$. Adicionalmente, con el SF36 tiene un $r=0,72$ para la dimensión física (DF) y $r=0,74$ para la dimensión emocional (DE); un alfa de Cronbach entre 0,81 a 0,95 y un coeficiente de 
correlación intraclase de 0,8913. Fue traducida en España y tuvo un coeficiente alfa de Cronbach $>0,8$, con coeficientes de correlación intraclase de 0,74 a 0,83 y puntajes diferentes de acuerdo con las clases funcionales $p<0,001^{14}$. En Colombia no existen instrumentos válidos para evaluar la calidad de vida en pacientes con falla cardiaca. Recientemente se publicó un artículo en el que se presentaron los resultados de la aplicación MLHFQ en un grupo de pacientes, con el objetivo de analizar la utilidad de este cuestionario en el seguimiento clínico y establecer la relación con la clase funcional y la fracción de eyección ${ }^{15}$.

Este estudio tuvo como objetivo hacer un proceso de validación prospectivo a partir de la escala original, traducir, retrotraducir y evaluar la validez, la confiabilidad y la sensibilidad al cambio en pacientes con falla cardiaca de un programa de rehabilitación cardíaca de un hospital de alta complejidad en Colombia.

\section{Materiales y métodos}

Este fue un estudio observacional de validación de una escala, prospectivo en tres momentos. En el primero se hizo la traducción, la prueba piloto y la adaptación cultural. En el segundo se evaluó la consistencia interna, la confiabilidad, así como la validez de contenido, constructo y criterio convergente y divergente. $Y$ en el tercero, longitudinal, se evaluó la sensibilidad al cambio.

Se utilizaron los criterios de la metodología COSMIN (Consensus based Standards for the selection of health status Measurement Instruments) ${ }^{16}$.

Se solicitó permiso al autor y se adquirieron la escala y las instrucciones para su recolección y calificación. El estudio se llevó a cabo en una institución de alta complejidad en Medellín, Colombia. La recolección de la información se hizo desde octubre de 2013 hasta septiembre de 2016. Se siguieron los lineamientos de la declaración de ética médica de Helsinki y según la resolución 8430 de 1993 de la república de Colombia se considera un estudio de riesgos mínimos. Fue aprobado por el comité de ética de La Clínica Las Américas, acta 72 del 5 de agosto de 2013. Los pacientes recibieron información respecto del objetivo del estudio y firmaron un consentimiento informado.

Se incluyeron 134 pacientes con diagnóstico de falla cardiaca sistólica y diastólica en clase funcional I-IV de la NYHA (New York Heart Association) y fracción de eyección $\leq 50 \%$. Se excluyeron menores de 16 años, que no aceptaron participar o con falla cardiaca aguda reversible, enfermedad grave concomitante, o quienes no tenían capacidad para completar el cuestionario en español. Los datos fueron recolectados por profesionales del área de la salud quienes recibieron entrenamiento. Dos médicos fisiatras con experiencia en rehabilitación cardíaca, una terapeuta física especialista en rehabilitación cardiaca y una terapeuta ocupacional efectuaron la clasificación y las pruebas funcionales e hicieron el programa de rehabilitación cardiaca.

Se utilizó un formulario con datos demográficos y clínicos. El cuestionario de calidad de vida de falla cardiaca, MLHFQ desarrollado por Rector y Cohn en 1984, auto-administrado, que consta de 21 ítems y dos dimensiones: física ( 8 ítems), emocional ( 5 ítems), otros ( 8 ítems). Respuestas en una escala tipo likert de 6 puntos de 0 "no limitación" a 5 "mucha limitación". El puntaje se obtuvo al sumar las respuestas, de 0 a 105 (peor calidad de vida), DF de 0-40 y DE de 0-25; los demás ítems suman $40^{11,14}$.

Se aplicó el SF 36, escala genérica validada en Colombia ${ }^{10}$, utilizada en investigaciones en pacientes con falla cardiaca ${ }^{17,18}$. Contiene 36 preguntas que miden los siguientes dominios: función física (FF), desempeño físico (DF), dolor corporal (DC), salud general (SG), vitalidad (VT), función social (FS), desempeño emocional (DE) y salud mental (SM). El resultado total va de 0-100 puntos (mejor es el estado de salud $)^{19}$.

El índice de estado de actividad de Duke - DASI, tiene un dominio y 12 ítems; cada ítem tiene un peso ponderado según el consumo de energía de la actividad evaluada. La puntuación total entre 0 (peor estado funcional) a 58,2 (mejor estado funcional) ${ }^{20,21}$.

Así mismo, se aplicó el World Health Organization Disability Assessment Schedule (WHO DAS II), instrumento genérico de medida de la discapacidad compuesto por 36 ítems y 6 dominios. El puntaje va desde 0 sin discapacidad a 100 mayor discapacidad ${ }^{22}$.

\section{Traducción y adaptación cultural}

El proceso de adaptación cultural y validez lingüística del cuestionario de Minnesota se desarrolló en dos etapas. La primera correspondió al proceso de traducción de la escala original al español hecha por dos traductores de forma independiente, quienes después de revisar y analizar las dos versiones las sintetizaron en una. Luego se hizo la retrotraducción del español al inglés con el fin de compararlas con la escala original. En la segunda fase, se hizo una prueba piloto con 15 pacientes y se evaluó la comprensión semántica de la escala.

\section{Validez de contenido}

Se incluyeron 134 pacientes, 5 por ítem para un total de 104 más 30\% de pérdidas. Para la valoración del ajuste del modelo se utilizó el "Root Mean Square Error of Approximation"' (RMSEA) que debe ser inferior a 0,07, el "Comparative fit index" (CFI) y el Tucker-Lewis index (TLI) que deben ser superiores a $0,90^{23,24}$. Este análisis se llevó a cabo con el software MPLUS versión $7.4^{25}$.

En la validez de constructo se compararon los resultados de las clases funcionales I -II vs. III-IV de la NYHA; para el cálculo del tamaño de la muestra se utilizó el software de la Pontificia Universidad Javeriana ${ }^{26}$, con los siguientes datos: error tipo I de 0,05, error tipo II 0,2, promedios del primero y segundo grupo de 13,2 y 22,9 , respectivamente, diferencia entre el promedio de los grupos de 9,7, DE de 12,1 para una hipótesis de dos colas, 55 por grupo más $10 \%$ de posibles pérdidas para un total de 121 pacientes. Se utilizó U Mann-Whitney para la comparación de grupos.

La validez de criterio convergente comparó la DF del MLHFQ con la FF y DF del SF 36, la DE del MLHFQ con la $S M$ y DE del SF 36. La validez divergente comparó la DF del MLHFQ con la de SM y DE del SF-36 y la DE del MLHFQ con la FF y DF del SF 36. El tamaño de muestra según estos datos fue el siguiente: error tipo I de 0,05, error tipo II de 0,2, coeficiente para la hipótesis nula de 0,4 , coeficiente para la 
hipótesis alterna de 0,6 más $10 \%$ de pérdidas para un total de 95 pacientes. El análisis estadístico se realizó por la matriz de correlaciones de Spearman.

\section{Proceso de fiabilidad}

La consistencia interna se aplicó a 134 pacientes; se evaluó la correlación entre los ítems y la escala con el coeficiente del alfa de Cronbach. La fiabilidad inter e intra observador se calculó de acuerdo con estos criterios: error tipo I de 0,05 , error tipo II 0,2, CCl para la hipótesis nula de 0,6 y la alterna de 0,8 más el $10 \%$ por pérdidas para un total de 44 pacientes.. En la fiabilidad intraobservador se aplicó el cuestionario MLHFQ en un primer momento y 15 días después, en la interevaluador en un período inferior a 48 horas. El análisis se realizó por medio del $\mathrm{CCl}$. Todos los análisis estadísticos se realizaron en SPSS 20.0.

Para evaluar la sensibilidad al cambio se hizo seguimiento al primero, tercero y sexto mes. El tamaño de la muestra se calculó así: error tipo I de 0,05, DE 19,8 media poblacional 8,22 para un tamaño de 63 , más un $10 \%$ por pérdidas para un total de 70 pacientes. El análisis estadístico se hizo por medio de la prueba de Wilcoxon. Se calculó el tamaño del efecto de Cohen; un tamaño del efecto de 0,8 es grande, 0,5 moderado y 0,2 bajo $^{27}$. Se calculó el mínimo cambio detectable (MCD), el cual se deriva del error estándar de la media (EEM), se calcula con la siguiente fórmula: $E E M=$ $D E_{\text {basal }} x \sqrt{1-R}$; donde $D E_{\text {basal }}$ es la desviación estándar de

Tabla 1 Características demográficas y clínicas según clase funcional de la New York Heart Association

\begin{tabular}{|c|c|c|c|c|}
\hline \multirow[t]{2}{*}{ Variable } & \multicolumn{3}{|c|}{ Clase funcional NYHA } & \multirow{2}{*}{$\begin{array}{l}\text { Total } \\
\mathrm{n}=134\end{array}$} \\
\hline & $I(n=32)$ & II $(n=86)$ & III $(n=16)$ & \\
\hline \multicolumn{5}{|l|}{ Género* } \\
\hline Masculino & $21(65,6)$ & $61(70,9)$ & $8(50)$ & $90(67,2)$ \\
\hline Edad & $62,2(10,4)$ & $64,3(9,5)$ & $70,2(9,0)$ & $64,5(9,9)$ \\
\hline \multicolumn{5}{|l|}{ Seguridad Social ${ }^{*}$} \\
\hline Contributivo & $32(100)$ & $84(97,7)$ & $15(93,8)$ & $131(97,8)$ \\
\hline \multicolumn{5}{|l|}{ Entidad } \\
\hline EPS & $28(87,5)$ & $81(93,1)$ & $15(93,8)$ & $124(91,9)$ \\
\hline \multicolumn{5}{|l|}{ MET $^{*}$} \\
\hline $1-4,9$ & $5(15,6)$ & $27(31,4)$ & $9(56,3)$ & $41(30,6)$ \\
\hline $5-7$ & $16(50,0)$ & $38(44,2)$ & $6(37,5)$ & $61(44,8)$ \\
\hline$>7$ & $11(34,4)$ & $21(24,4)$ & $1(6,3)$ & $33(24,6)$ \\
\hline \multicolumn{5}{|l|}{ Diagnóstico* } \\
\hline Enfermedad coronaria & $25(78,1)$ & $60(68,5)$ & $10(62,5)$ & $94(70,1)$ \\
\hline Valvular & $1(3,1)$ & $7(8,1)$ & $2(12,5)$ & $10(7,5)$ \\
\hline Otros & $6(18,8)$ & $20(23,3)$ & $4(25)$ & $30(22,4)$ \\
\hline \multicolumn{5}{|l|}{ Fracción de eyección } \\
\hline $40-50$ & $16(50)$ & $25(29,1)$ & $3(18,8)$ & $44(32,8)$ \\
\hline$<40$ & $16(50)$ & $61(70,9)$ & $13(81,3)$ & $90(67,2)$ \\
\hline \multicolumn{5}{|l|}{ Reintegro ocupacional" } \\
\hline Parcial & $17(53,1)$ & $46(53,5)$ & $7(43,8)$ & $70(52,2)$ \\
\hline Reintegro completo & $4(12,5)$ & $6(7)$ & $2(12,5)$ & $12(9,0)$ \\
\hline Reubicado & $0(0)$ & $1(1,2)$ & $0(0)$ & $1(0,7)$ \\
\hline DASI $(0-58,2)^{* *}$ & $19,6 \pm 11,2$ & $14,8 \pm 8,8$ & $13 \pm 8,8$ & $15,8 \pm 9,6$ \\
\hline \multicolumn{5}{|l|}{ MLHFQ $^{* *}$} \\
\hline Dimensión física (0-40) & $7,9 \pm 7,4$ & $9,8 \pm 8,0$ & $12,6 \pm 8,2$ & $9,7 \pm 7,9$ \\
\hline Dimensión emocional (0-25) & $4,0 \pm 4,3$ & $4,9 \pm 4,8$ & $5,8 \pm 4,7$ & $4,8 \pm 4,7$ \\
\hline Dimensión otros $(0-45)$ & $9,3 \pm 6,1$ & $11,2 \pm 7,6$ & $11,1 \pm 6,4$ & $10,8 \pm 7,1$ \\
\hline Total (0-105) & $21,3 \pm 15,1$ & $26,0(17,3)$ & $29,4 \pm 15,7$ & $25,3 \pm 16,7$ \\
\hline \multicolumn{5}{|l|}{ SF-36 $(0-100)^{* *}$} \\
\hline Función física & $70,3 \pm 18,4$ & $57,8 \pm 21,4$ & $57,2 \pm 24,8$ & $60,7 \pm 21,7$ \\
\hline Desempeño físico & $50,6 \pm 30,8$ & $39,5 \pm 30,0$ & $41,5 \pm 38,6$ & $42,3 \pm 31,4$ \\
\hline WHO-DAS $(0-100)^{* *}$ & $18,9 \pm 17,7$ & $12,6 \pm 12,4$ & $14,7 \pm 12,8$ & $14,7 \pm 13,9$ \\
\hline
\end{tabular}

" Las variables cualitativas se citan en medida de frecuencias.

** Las variables cuantitativas con promedios y desviación estándar. NYHA: New York Heart Association; EPS: entidad promotora de salud; MET: equivalentes metabólicos; DASI: Índice de estado de actividad de Duke, MLHFQ: Minnesota Living With Heart Failure Questionnaire; SF-36; cuestionario de calidad de vida relacionada con la salud; WHO-DAS: World Health Organization Disability Assessment Schedule. 


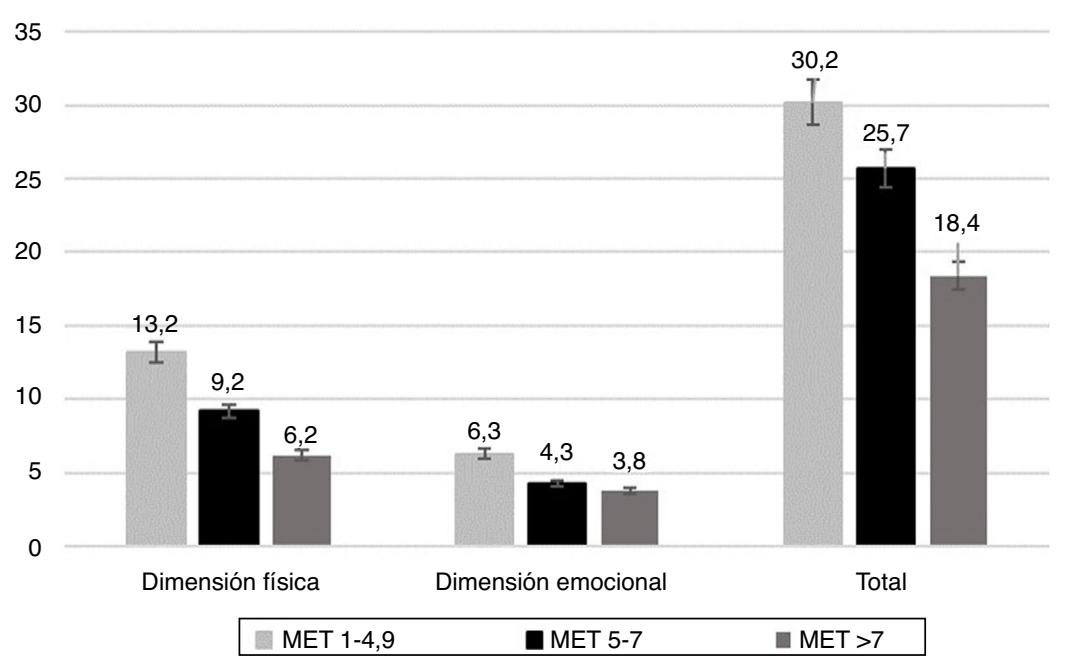

Figura 1 Promedios de las dimensiones física y emocional y total del MLHFQ de acuerdo con los MET y el valor p: DF: 0,01, DE: 0,037 y total: 0,015. Las barras representan el intervalo de confianza del $95 \%$.

Tabla 2 Comparación de los resultados del MLHFQ según clase funcional NYHA I, II, II

\begin{tabular}{llll}
\hline & Media (DE.) & Diferencia de medias Error estándar & Valor de $\mathrm{p}$ \\
\hline NYHA I vs. NYHA II & $21,25(15,15)$ vs $25,66(17,30)$ & $-4,73(3,47)$ & 0,165 \\
NYHA II vs. NYHA III & $25,66(17,30)$ vs $29,44(15,74)$ & $-3,45(4,65)$ & 0,846 \\
NYHA I vs. NYHA III & $21,25(15,15)$ vs $29,44(15,74)$ & $-8,19(4,70)$ & 0,405 \\
\hline
\end{tabular}

NYHA: New York Heart Association, DE = desviación estándar.

la medición inicial y $\mathrm{R}$ el coeficiente de fiabilidad intraevaluador. El MCD se calcula de la siguiente manera: EEM $\mathrm{x}$ $z$-score $\times \sqrt{2}$. El $z$-score se asumió para un valor del $95 \%$ de confianza.

\section{Resultados}

\section{Características sociodemográficas y clínicas}

Se incluyeron 134 pacientes, de los cuales el 67,2\% (90) fueron hombres; la edad promedio fue de 64,5 DE: 9,9 años. 55 participantes $(40,7 \%)$ eran empleados y 48 (35,6\%) pensionados. Se encontró índice de masa corporal (IMC) normal en 54 (40,0\%), sobrepeso en 52 (38,5\%), diabetes en $34(25,2 \%)$, dislipidemia en 104 (77,0\%) y complicaciones osteomusculares en $15(11,1 \%) ; 69(51,1 \%)$ realizaban actividad física. En el $70,1 \%$ de los pacientes la etiología de la falla cardiaca fue la enfermedad coronaria (tabla 1).

\section{Traducción y adaptación cultural}

El primer cambio fue con relación al tiempo gramatical; la primera parte del cuestionario original se encuentra en presente progresivo del verbo "ser o estar"'. El grupo de adaptación decidió que el tiempo sería pretérito perfecto simple para todo el cuestionario. Se hicieron cambios al párrafo introductorio y a algunas palabras para facilitar la comprensión (anexo1).

\section{Validez}

El modelo mostró un ajuste adecuado con un valor de Chi cuadrado $=203,8, \mathrm{gl}=186 ; \mathrm{p}=0,1766$. Los valores de CFI (Comparitive Fit Index) fueron de 0,97, TLI=0,96, y RMSEA $=0,027$. En el anexo 2 se observa que el modelo se ajusta bien a las tres dimensiones e ítems propuestos por los autores ${ }^{11}$.

En la validez de constructo se observó que el puntaje fue mejor a medida que se optimizó la capacidad funcional medida en MET (fig. 1). El puntaje total del MLHFQ en la clase funcional I fue de 21,3 (DE:15,1), en la clase funcional II fue de 26,0 (DE:17,3) y en la clase funcional III fue de 29,4 $(\mathrm{DE}: 16,7)$. El hecho de no encontrar diferencias significativas puede explicarse por el tamaño de la muestra en el grupo de la clase funcional III (tabla 2).

La validez convergente entre la DF del MLHFQ y el SF36 obtuvo una correlación para la FF de $-0,681$ y para el DF 0,524 esta correlación fue moderada. Entre la DE del MLHFQ y la SM del SF36 se encontró $-0,698$, con la VT $-0,636$, se esperaban valores superiores a $-0,4$. La validez divergente de la DF del MLHFQ con el DE y la SM del SF-36 encontró valores de $-0,314$ y $-0,470$ respectivamente. En la DE del MLHFQ con la FF y DF del SF36 valores de $-0,321$ y $-0,499$ respectivamente; en ambos casos se esperaba un valor < 0,4 . Todos los valores fueron estadísticamente significativos con un valor de $\mathrm{p}<0,05$ (tabla 3).

Se probó la validez de criterio con WHODASII y DASI. Las correlaciones más altas fueron de la DF del MLHFQ y la 
Tabla 3 Validez de criterio convergente y divergente entre el MLHFQ y el SF-36, WHO-DAS y el DASI

\begin{tabular}{|c|c|c|c|c|}
\hline & \multirow[t]{2}{*}{ Dominios } & \multicolumn{3}{|c|}{ MLHFQ } \\
\hline & & $\begin{array}{l}\text { Dimensión física } \\
\text { Correlación } \\
\text { de Spearman }\end{array}$ & $\begin{array}{l}\text { Dimensión emocional } \\
\text { Correlación } \\
\text { de Spearman }\end{array}$ & $\begin{array}{l}\text { Total } \\
\text { Correlación } \\
\text { de Spearman }\end{array}$ \\
\hline \multirow[t]{8}{*}{ SF-36 } & Función física & $-0,681^{* *}$ & $-0,321^{* *}$ & $-0,622^{* *}$ \\
\hline & Desempeño físico & $-0,524^{* *}$ & $-0,499^{* *}$ & $-0,668^{* *}$ \\
\hline & Desempeño emocional & $-0,314^{* *}$ & $-0,307^{* *}$ & $-0,302^{* *}$ \\
\hline & Salud mental & $-0,470^{* *}$ & $-0,698^{* *}$ & $-0,566^{* *}$ \\
\hline & Dolor corporal & $-0,459^{* *}$ & $-0,277^{* *}$ & $-0,464^{* *}$ \\
\hline & Función social & $-0,428^{* *}$ & $-0,376^{* *}$ & $-0,493^{* *}$ \\
\hline & Salud general & $-0,435^{* *}$ & $-0,365^{* *}$ & $-0,415^{* *}$ \\
\hline & Vitalidad & $-0,670^{* *}$ & $-0,636^{* *}$ & $-0,701^{\text {*** }}$ \\
\hline \multirow[t]{8}{*}{ WHO-DAS } & Comprensión y comunicación & $0,345^{* *}$ & $0,408^{* *}$ & $0,384^{* *}$ \\
\hline & Capacidad para moverse a su alrededor & $0,560^{* *}$ & $0,303^{* *}$ & $0,512^{* *}$ \\
\hline & Cuidado personal & $0,444^{* *}$ & $0,270^{* *}$ & $0,441^{\text {** }}$ \\
\hline & Relaciones con otras personas & $0,280^{* *}$ & $0,237^{* *}$ & $0,290^{* *}$ \\
\hline & Actividades de la vida diaria & $0,525^{* *}$ & $0,340^{* * *}$ & $0,496^{* *}$ \\
\hline & Actividades laborales & $0,546^{* * *}$ & $0,345^{*}$ & $0,536^{* *}$ \\
\hline & Participación en sociedad & $0,501^{* *}$ & $0,451^{* *}$ & $0,540^{* *}$ \\
\hline & Puntuación global WHO-DAS & $0,612^{* *}$ & $0,361^{*}$ & $0,590^{* *}$ \\
\hline DASI & Dimensión física & $-0,594^{* *}$ & $-0,326^{* *}$ & $-0,519^{* *}$ \\
\hline
\end{tabular}

MLHFQ: Minnesota Living With Heart Failure Questionnaire; SF-36: cuestionario de calidad de vida relacionada con la salud; WHO-DAS: World Health Organization Disability Assessment Schedule; DASI: Índice de estado de actividad de Duke

** Significativo $\mathrm{p}<0,001$. Coeficientes de correlación de Spearman.

capacidad de moverse a su alrededor del WHODASII con un valor de 0,560 , con el dominio de actividades de la vida diaria 0,525 y con la puntuación global de 0,612 . La correlación entre el dominio de DF de MLHFQ y el DASI fue moderada, $-0,519$.

\section{Consistencia interna y fiabilidad intra- e interobservador}

La consistencia interna entre los ítems y la escala mostró una homogeneidad alta $(0,87)$ medida con alfa de Cronbach. La fiabilidad intraobservador medida con el CCI para el puntaje total fue de 0,91 (IC 95\% 0,84-0,96) y la interevaluador para el puntaje total fue de 0,94 $(0,90-0,97)$ (tabla 4).

Se encontraron diferencias estadísticamente significativas entre el inicio y el primer, tercer y sexto mes en todas las dimensiones y con el puntaje total. Se calculó el tamaño del efecto de Cohen y se encontró para el puntaje total un efecto moderado $(-0,4)$ al primer mes; y grande el tercero y sexto meses $-0,84$ (tabla 5). Por último, el mínimo cambio detectable $(M C D)$ para la DF fue de 6,0 , para la DE de 4,8 y para el total de 12,6.

Discusión

En la primera fase se hizo el proceso de traducción y retrotraducción y se obtuvo una adaptación cultural del instrumento. La validez de contenido identifica las dimensiones descritas por los autores de la escala ${ }^{14}$. En la validación española del MLHFQ para atención primaria con un análisis exploratorio se confirmó la estructura de dos dominios ${ }^{28}$. En la validez de constructo al comparar los puntajes de acuerdo con las clases funcionales NYHA se encontró que el puntaje empeoró a medida que aumentó el compromiso funcional; sin embargo, la diferencia no fue significativa, debido a que la recolección de la muestra se hizo en forma consecutiva y no estratificada y hubo pocos pacientes en el estrato III. En los estudios de Garin et al. y Naviero, estas diferencias fueron significativas ${ }^{12,28}$. Por su parte, en un estudio hecho en Colombia se encontraron diferencias significativas de acuerdo con la clase funcional en el DF, pero no en el emocional y también hubo pocos pacientes en la clase funcional $\mathrm{III}^{15}$. La calidad de vida fue mejor en los pacientes que tuvieron pruebas funcionales mayores de 7 MET y esta diferencia fue significativa.

Se encontró una validez de criterio adecuada al compararlo con el SF36, con el WHO-DAS II o con el DASI. LoS coeficientes de correlación de Spearman superaron - 0,4 en los dominios que correspondían a un mismo constructo y estuvieron por debajo en los constructos diferentes. Esto es similar a las validaciones hechas en España; en la de Garin las correlaciones convergentes fueron superiores a 0,52 y las divergentes $<0,5^{12}$. En la validación de Naviero las convergentes estuvieron entre $-0,43$ a $-0,73$ y las divergentes entre $-0,33$ a $-0,51^{28}$. Tuvo una correlación alta con El DASI ${ }^{29}$. Este es el primer trabajo en el que el MLHFQ se compara con el WHODAS II que es la escala derivada de la CIF recomendada por la OMS para medir el funcionamiento humano ${ }^{22}$. La DF del MLHFQ tuvo muy buena correlación con las escalas que miden el FF y con el puntaje global y el dominio de participación, lo cual es muy importante pues muchas veces las escalas de calidad de vida no tienen en cuenta el componente social.

En la fiabilidad se encontró una buena consistencia interna $(0,87)$ que indica que se está evaluando un solo constructo. En el estudio de Rector et al. ${ }^{11}$ se encontró 0,94, en el de Gorkin 0,95 y en el de Bennett de 0,95 
Tabla 4 Fiabilidad intra e inter observador de las dimensiones del MLHFQ

\begin{tabular}{lll}
\hline & $\begin{array}{l}\text { Intraobservador } \\
\left(\mathrm{CCI}^{*} ; \mathrm{IC} 95 \%\right)\end{array}$ & $\begin{array}{l}\text { Interobservador } \\
\left(\mathrm{CCl}^{*} ; \mathrm{IC} 95 \%\right)^{-}\end{array}$ \\
\hline Dimensión física & 0,$92 ;(0,85-0,95)$ & 0,$93 ;(0,88-0,96)$ \\
Dimensión emocional & 0,$85 ;(0,75-0,93)$ & $0,86(0,75-0,92)$ \\
Otros & 0,$87 ;(0,75-0,94)$ & $0,89(0,80-0,93)$ \\
Total & 0,$91 ;(0,84-0,96)$ & $0,94(0,90-0,97)$ \\
\hline
\end{tabular}

" CCI: coeficiente de correlación intraclase, IC: intervalo de confianza del 95\%.

Tabla 5 Sensibilidad al cambio y tamaño del efecto de las dimensiones del MLHFQ a 1,3 y 6 meses de seguimiento

\begin{tabular}{|c|c|c|c|c|c|c|c|}
\hline \multirow[t]{2}{*}{ Dimensión } & \multirow{2}{*}{$\begin{array}{l}\text { Inicial } \\
\text { Media (DE) }\end{array}$} & \multicolumn{2}{|c|}{ Primer mes } & \multicolumn{2}{|c|}{ Tercer mes } & \multicolumn{2}{|c|}{ Sexto mes } \\
\hline & & Media (DE) & $\mathrm{TE}^{\bullet}$ & Media (DE) & $\mathrm{TE}$ & Media (DE) & TE \\
\hline Física & $8,71(7,45)$ & $6,39(6,73)^{* * * *}$ & $-0,33$ & $4,63(5,71)^{* * *}$ & $-0,61$ & $4,54(5,98)^{* * *}$ & $-0,63$ \\
\hline Emocional & $4,09(4,20)$ & $3,45(3,88)^{*}$ & $-0,16$ & $2,57(3,07)^{* *}$ & $-0,31$ & $2,50(2,85)^{* *}$ & $-0,39$ \\
\hline Otros & $7,90(6,34)$ & $6,08(4,89)^{* * * *}$ & $-0,64$ & $4,31(3,96)^{* * * *}$ & $-1,03$ & $4,55(4,34)^{* * * *}$ & $-0,99$ \\
\hline Total & $22,51(14,92)$ & $15,92(13,21)^{* * * *}$ & $-0,47$ & $11,51(10,18)^{* * *}$ & $-0,84$ & $11,59(11,16)^{* * *}$ & $-0,84$ \\
\hline
\end{tabular}

- TE: Tamaño del efecto no paramétrico. Se aplicó la prueba de Wilcoxon y se obtuvieron valores $p$ así: Valor $p$

* $p<0,05$

** $p<0,01$;

***; $p<0,001$.

La fiabilidad intraobservador fue alta $(0,84$ a 0,96$)$; en la validación española fue $>0,7^{14}$, mientras que en el estudio de Bennett fue de 0,87. En el estudio de los autores se encontró muy buena fiabilidad interevaluador, entre 0,86 a 0,94 , aspecto que no fue valorado en otros estudios y que es importante en algunas culturas en las que se debe aplicar el instrumento por entrevista por diferentes evaluadores.

Una fortaleza de este estudio es que demostró buena sensibilidad al cambio, el tamaño del efecto en los meses tercero y sexto fue grande, en el puntaje total $(-0,84)$, moderado en la DF $(0,63)$ y pequeño en la DE. En el trabajo de Garin fueron cercanos a 0,4 $4^{14}$. La calidad de vida entre los meses tercero y sexto fue muy similar; el cambio mayor se presentó entre los meses primero y tercero.

De acuerdo con lo descrito por Rector un cambio en el puntaje individual necesita ser mayor a 2,77 veces el error de la medida ${ }^{31}$. El MCD calculado fue de 12,6. Se ha propuesto que 5 puntos en el puntaje global es un cambio clínicamente importante en un individuo ${ }^{14}$.

Esta escala evalúa la percepción de los pacientes que ha sido promovida por el proyecto PROMIS (Patient Report Outcomes Measurement Information System) ${ }^{32}$. Se convierte en un desenlace que puede evaluarse como primario, secundario o compuesto en los diferentes ensayos clínicos. $\mathrm{Ha}$ sido utilizado en estudios de enfermedad arterial coronaria, terapia de revascularización, falla cardíaca crónica, fibrilación auricular, terapia de resincronización, cardiodesfibriladores, cirugía cardiovascular y otros dispositivos. En algunos de estos estudios se ha demostrado mejoría hasta más de 30 puntos del MHLFQ; sin embargo, hay otros estudios en los que una intervención no ha mostrado la mejoría en la calidad de vida esperada ${ }^{33}$.
La principal limitación de este estudio fue la selección de pacientes, que se hizo de manera consecutiva y no estratificada por lo cual no hubo pacientes NYHA IV y el 64,4\% de la muestra fue NYHA II, que corresponde a la población que se atiende en el sitio en el que se recolectó la muestra. A los pacientes no se les hicieron pruebas con consumo de oxígeno, pero se les practicó la prueba de esfuerzo submáxima, que es una medida objetiva válida del estado funcional con la medición de los MET. Otra limitación pudiera ser que los pacientes corresponden a una sola institución y sería importante tener pacientes de diferentes regiones geográficas.

\section{Conclusión}

El cuestionario de Minnesota de calidad de vida para pacientes con falla cardíaca (MLHFQ) es válido y confiable para utilizar en población colombiana y posee propiedades psicométricas adecuadas en la mayoría de los procesos de validez, fiabilidad y sensibilidad al cambio. Adicionalmente, este instrumento validado servirá para ampliar la información de sus características psicométricas y su interpretación clínica para futuros estudios mundiales.

\section{Reconocimientos y agradecimientos}

A quienes participaron en la recolección de los datos, a la enfermera Rocío Romero y la fisioterapeuta Deisy Sierra. A la clínica Las Américas y a los profesionales del área de Rehabilitación cardíaca por su disposición para participar en esta investigación. 


\section{Financiación}

Esta investigación fue realizada con recursos de sostenibilidad de la Universidad de Antioquia 2016-2017 y recursos en especie y logísticos de la Clínica Las Américas.

\section{Conflicto de intereses}

Ninguno.

\section{Anexo. Material adicional}

Se puede consultar material adicional a este artículo en su versión electrónica disponible en doi:10.1016/j.rccar. 2019.04.003.

\section{Bibliografía}

1. Ministerio de Salud y Protección Social, Departamento Administrativo de ciencia, Tecnología e Innovación-Colciencias. Guía de práctica clínica para la prevención, diagnóstico, tratamiento y rehabilitación de la falla cardíaca en población mayor de 18 años B, C y D. Guía para profesionales de la Salud 2016.

2. Colvin M, Sweitzer NK, Albert NM, Krishnamani R, Rich MW, Stough WG, et al. Heart Failure in Non-Caucasians, Women, and Older Adults: A White Paper on Special Populations From the Heart Failure Society of America Guideline Committee. J Card Fail. 2015;21:674-93.

3. Sociedad Colombiana de Cardiología y Cirugía Cardiovascular. Consenso Colombiano para el diagnóstico y tratamiento de la insuficiencia cardíaca crónica. Primera edición. Noviembre 2014. Disponible en: http://scc.org.co/consenso-colombianopara-el-diagnostico-y-tratamiento-de-la-insuficienciacardiaca-aguda-y-cronica/.

4. Sánchez-Ropero EM, Vera-Giraldo CY, Navas-Ríos CM, OrtizRangel SD, Rodríguez-Guevara C, Vargas-Montoya DM, et al. Validación para Colombia del cuestionario para la "Medición de la capacidad funcional en pacientes con falla cardíaca'. Rev Colomb Cardiol. 2018;25:356-65.

5. Brock D. Medidas de calidad de vida en el cuidado de la salud y la ética médica. En: Nussbaum MC, Sen A, compiladores. La calidad de vida. México, D. F.: The United Nations University, Fondo de Cultura Económica; 1998. p. 135-81.

6. Organización Mundial de la Salud, Grupo sobre la Calidad de Vida. ¿Qué calidad de vida? Foro Mundial de la Salud. 1996;17(4):385-87.

7. Riegel B, Moser DK, Anker SD, Appel LJ, Dunbar SB, Grady KL, et al. State of the science: promoting self-care in persons with heart failure. Circulation. 2009;120:1141-63.

8. Snyder CF, Jensen R, Segal J, Wu A. Patient-Reported Outcomes (pros): putting the patient perspective in patient-centered outcomes research. Med Care. 2013;51: S73-9.

9. Szyguła-Jurkiewicz B, Zakliczyński M, Owczarek A, Partyka R, Mościński M, Pudlo R, et al. Low health-related quality of life is a predictor of major adverse cardiovascular events in patients with chronic non ischemic Heart Failure. Kardiochir Torakochirurgia Pol. 2014;11:283-8.

10. Lugo LH, García HI, Gómez C. Confiabilidad del cuestionario de calidad de vida en salud SF-36 en Medellín, Colombia. Rev Fac Nac Salud Pública. 2006;24:37-50.

11. Rector TS, Kubo SH, Cohn JN. Patient's self-assessment of their congestive heart failure: Content, reliability and validity of a new measure: The Minnesota living with heart failure questionnaire. Heart Fail. 1987;3:198-219.

12. Garin O, Ferrer M, Pont A, Rue M, Kotzeva A, Wiklund I, et al. Disease-specific health-related quality of life questionnaires for heart failure: A systematic review with metaanalyses. Qual Life Res. 2008; 18:71-85.

13. Tate CWI, Robertson AD, Zolty R, Shakar SF, Lindenfeld J, Wolfel EF, et al. Quality of life and prognosis in heart failure: Results of the beta-blocker evaluation of survival trial (BEST). J Card Fail. 2007;13:732-7.

14. Garin O, Soriano N, Ribera A, Ferrer M, Pont A, Alonso J, et al. Validación de la versión española del Minnesota Living with Heart Failure Questionnaire (MLHFQ). Rev Esp Cardiol. 2008;61:251-9.

15. Palomino G, Pareja MJ, Pareja MC, Nevado N, Mendoza F, Dávila FA, et al. Utilidad de la Escala de Minnesota en el seguimiento de los pacientes con insuficiencia cardíaca crónica. Cardiocore. 2018;53:55-60.

16. Mokkink L, Terwee C, Patrick D, Alonso J, Stratford P, Knol D, et al. The COSMIN checklist for assessing the methodological quality of studies on measurement properties of health status measurement instruments: an international Delphi study. Quality Life Res. 2010;19:539-49.

17. Rodriguez-Artalejo F, Guallar-Castillon P, Pascual CR, et al. Health-related quality of life as a predictor of hospital readmission and death among patients with heart failure. Arch Intern Med. 2005; 165:1274-9.

18. Lugo LH, Navas CM, Plata JA, Ortiz SD, Caraballo D, Henao AC, et al. Ensayo clínico aleatorizado para evaluar el efecto de un programa de rehabilitación cardiaca supervisado con ejercicio en el consumo de oxígeno, la función y calidad de vida de pacientes con falla cardiaca crónica. Rev Colomb Cardiol. 2018;25:106-15.

19. García HI, Vera CY, Lugo LH. Calidad de vida relacionada con la salud en Medellín y su área metropolitana, con aplicación del SF-36. Rev Fac Nac Salud Pública. 2014;32:26-39.

20. Hlatky MA, Boineau RE, Higginbotham MB, Lee KL, Mark DB, Califf RM, et al. A brief self-administered questionnaire to determine functional capacity (the Duke Activity Status Index). Am J Cardiol. 1989;64:651-4.

21. Fan X, Lee KS, Frazier SK, Lennie TA, Moser DK. Psychometric testing of the Duke Activity Status Index in patients with heart failure. Eur J Cardiovasc Nurs. 2015;14:214-21.

22. Vázquez JL, Herrera S, Vázquez E, Gaite L. Cuestionario para la evaluación de discapacidad de la organización mundial de la salud. Versión Española de WHO Disability Assessment Schedule II. Madrid. 2006:197.

23. Hu LT, Bentler PM. Cutoff criteria for fit indexes in covariance structure analysis: Conventional criteria versus new alternativesStructural Equation Modeling: A Multidisciplinary Journal;. 1999:1-55.

24. Streiner DL, Norman, Geoffrey R, Cairney J. Health Measurement Scales. 5 a. Ed Oxford Scholar; 2015.

25. Muthén LK, Muthén BO. Mplus User's Guide. $8^{\text {th }}$. Ed Los Angeles: Muthén \& Muthén; 2017.

26. Pérez A, Rodríguez MN, Gil JF RG. Tamaño de la muestra versión 1.1. Pontificia Universidad Javeriana. Unidad de Epidemiología Clínica [consultado Ene 2017]. Disponible en: http://hermes.javeriana.edu.co/tamamu.

27. Fritz CO, Morris PE, Richler JJ. Effect size estimates: Current use, calculations, and interpretation. J Exp Psychol Gen. 2012;141:2-18.

28. Naveiro-Rilo JC, Diez Juárez DM, Blanco AR, Rebollo-Gutiérrez F, Rodríguez-Martínez A, Rodríguez-García MA. Validación del Minnesota Living with Heart Failure Questionnaire en atención primaria. Rev Esp Cardiol. 2010;63:1419-27. 
29. Fan X, Lee KS, Frazier SK, Lennie TA, Moser DK. Psychometric testing of the Duke Activity Status Index in patients with Heart Failure. Eur J Cardiovasc Nurs. 2015;14:214-21.

30. Bennett SJ, Oldridge NB, Eckert GJ, Embree JL, Browning S, Hou N, et al. Comparison of quality of life measures in heart failure. Nurs Res. 2003;52:207-16.

31. Rector TS. A conceptual model of quality of life in relation to heart failure. J Card Fail. 2005;11:173-6.
32. Department of Health \& Human Services, U.S. PROMIS ${ }^{\circledR}$ : dynamic tools to measure health outcomes from the patient perspective [consultado Ene 2017]. Disponible en: http: //www.nihpromis.org.

33. Mark DB. Assessing quality-of-life outcomes in cardiovascular clinical research. Nat Rev Cardiol. 2016;13:286-308. 